\title{
Lidar measurement of atmospheric aerosol extinction profiles: a comparison between two techniques - Klett inversion and pure rotational Raman scattering methods
}

\author{
V. M. Mitev, I. V. Grigorov, and V. B. Simeonov
}

\begin{abstract}
Two lidar methods of determining an atmospheric extinction coefficient profile are compared. The methods are the Klett inversion method for elastic lidar return and the log-derivative method for rotational Raman backscattered signal processing. The comparison includes numerical modeling and processing of lidar measurements when both the elastic and the rotational Raman backscattered signals are measured simultaneously. The suggested idea is that such a comparison can be used as a criterion for the reliability of the results of lidar measurements, similar to the comparison between the results of lidar and contact measurements.
\end{abstract}

Key words: Lidar, aerosol extinction, Klett inversion method, rotational Raman scattering.

\section{Introduction}

Until now the Klett inversion method for elastic backscattered lidar return processing and its improvements has been considered a basic method for lidar determination of the atmospheric aerosol extinction profile. ${ }^{1-3}$ Because the concentration and other physical parameters of the aerosol component of the atmosphere fluctuate in an unpredictable way, there are two major setbacks associated with this method: first, the necessity of determining both the aerosol backscatter and the aerosol extinction coefficients from a single lidar return, i.e., from a single lidar equation; and second, the necessity of establishing boundary conditions or a reference value for the extinction coefficient $\alpha_{\text {ref }}$ in the far end of the measured profile. In the general practice of lidar measurements this necessity can be fulfilled only by $a$ priori information or reasonable assumptions, or, concerning $\alpha_{\text {ref }}$, by using estimations based on the same elastic lidar return. The Raman lidar return signal is determined by the molecular components of the atmosphere, and in the lower troposphere it is affected only by the aerosol extinction. Detection and processing the profile of this signal permits the

The authors are with the Institute of Electronics, Bulgarian Academy of Sciences, 72 Trakia Boulevard, Sofia 1784, Bulgaria.

Received 2 May 1991.

0003-6935/92/306469-06\$05.00/0.

( 1992 Optical Society of America. determination of the profile of the atmospheric extinction coefficient (see Refs. 4 and 5 and the references therein).

The possibility of determining the extinction coefficient from the pure rotational Raman scattering (RRS) lidar return follows from analysis of the RRS lidar equation. By taking the logarithm of the ratio of two RRS lidar returns $P(H)$ and $P(H+\Delta H)$ from two adjacent space samples, both with duration $\Delta H$ and placed at altitudes (distances) $H$ and $H+\Delta H$ respectively, we can write the extinction coefficient $\alpha(H)$ as in Ref. 4:

$$
\begin{aligned}
\alpha(H)= & \frac{1}{2 \Delta H}\left\{\ln \frac{P(H)}{P(H+\Delta H)}-\ln \frac{\sigma[T(H)]}{\sigma[T(H+\Delta H)]}\right. \\
& -\ln \frac{n(H)}{n(H+\Delta H)}-2 \ln \frac{H+\Delta H}{H}
\end{aligned}
$$

This treatment is called the log-derivative method. In Eq. (1) $\sigma[T(H)]$ is the molecular RRS cross section for nitrogen and oxygen, $n(H)$ is the molecular number density of nitrogen and oxygen, and $T(H)$ is the temperature. To obtain Eq. (1) we assume that the lidar overlap function does not depend on the distance $H$, that the values of the laser and the RRS wavelengths $\lambda_{0}$ and $\lambda_{R}$ are close, and that the extinction is determined essentially by the aerosol content if $\lambda_{0}$ is in the region of the visible spectrum. When a vibra- 
tional Raman line is detected, either from nitrogen or from oxygen (as in Ref. 5), the number 2 in the denominator of Eq. (1) is replaced by $1+\lambda_{0} / \lambda_{v}$ because of the wavelength dependence of the aerosol extinction coefficient; if molecular scattering is considered, this expression will be $1+\lambda_{0}{ }^{4} / \lambda_{v}{ }^{4} ; \lambda_{v}$ is the wavelength of the vibrational Raman line [see Eq. (7) of Ref. 5]. When analyzing these two methods, we find that questions appear about their advantages and disadvantages, and the compatibility of their results. The idea in this paper is to present a treatment of these questions based on numerical modeling and lidar experiments.

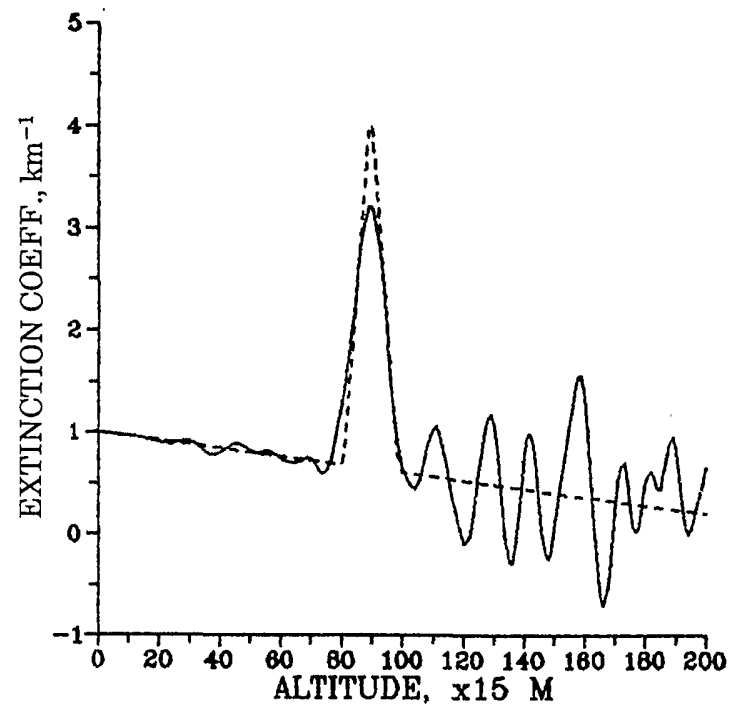

(a)

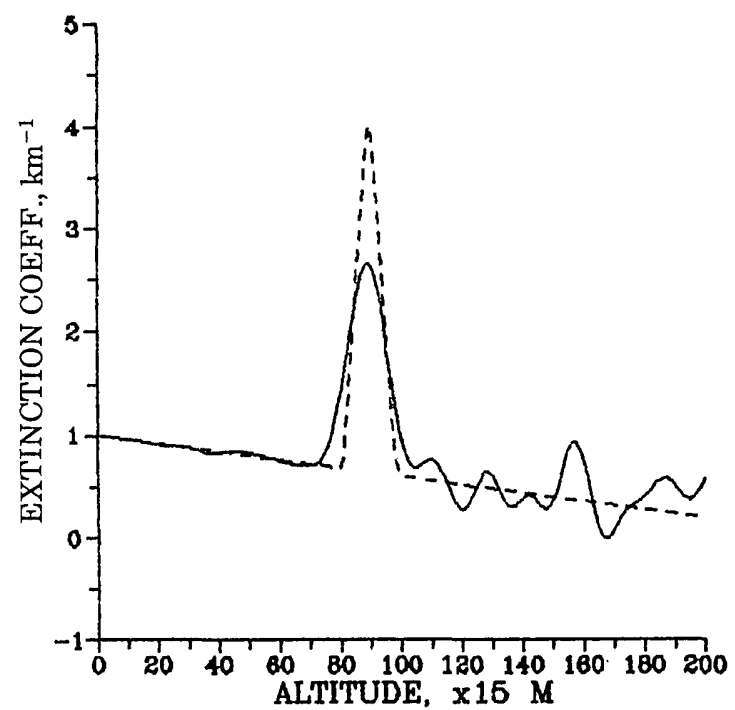

(b)

Fig. 1. (a) Numerical experiment: the model extinction coefficient (dashed curve), and the determined log-derivative extinction coefficient, derived with a twofold sliding average for the extinction coefficient (solid curve). (b) Numerical experiment: the model extinction coefficient (dashed curve) and the determined logderivative extinction coefficient, derived with a tenfold sliding average for the extinction coefficiont (solid curve).

\section{Numerical Experiment}

An initial comparison between the log-derivative and Klett methods is provided by numerical experiment and is carried out by assuming a lidar with a photoncounting detection system and a spatial resolution along the vertical profile of $15 \mathrm{~m}$ (space cell), and up to a $2-3-\mathrm{km}$ altitude. For such an altitude the linear distribution of the aerosol extinction coefficient is applied as a convenient approximation of the known exponential models. A denser layer serves as a perturbation for lidar backscattered signals and is positioned at an altitude of 90-100 space cells. The numerical modeling has the following sequence: from the initial model aerosol extinction coefficient $\alpha_{m}(H)$ the normalized $S$ functions for the RRS and aerosol backscattered signals are calculated. The normalized $S$ function for the model RRS lidar return is

$$
\begin{aligned}
S_{\mathrm{RRS}}(H)- & S_{\mathrm{RRS}}\left(H_{0}\right) \\
& =\ln \left\{\frac{\sigma[T(H)] n(H)}{\sigma\left[T\left(H_{0}\right)\right] n\left(H_{0}\right)}\right\}-2 \int_{H_{0}}^{H} \alpha_{m}(h) \mathrm{d} h .
\end{aligned}
$$

For standard atmosphere the factor $\sigma[T(H)] /$ $\sigma\left[T\left(H_{0}\right)\right]$ is close to 1 and can be neglected compared with the factor $n(H) / n\left(H_{0}\right)$. The normalized model $S$ function for the elastic backscatter (which is indicated by the subscript $E$ ) is ${ }^{1}$ :

$$
S_{E}(H)-S_{E}\left(H_{0}\right)=\ln \frac{\alpha_{m}(H)}{\alpha_{m}\left(H_{0}\right)}-2 \int_{H_{0}}^{H} \alpha_{m}(h) \mathrm{d} h .
$$

In Eqs. (2) and (3) $H_{0}$ is the fixed reference altitude at the near end of the profile. In Eq. (3) the relationship between the coefficients of backscattering $\beta$ and of extinction $\alpha$, i.e., $\beta=$ constant $\alpha^{\kappa}$ is adopted as valid, with constant lidar ratio and $\kappa=1$. Because of the Poisson statistics of the real observed lidar sig-

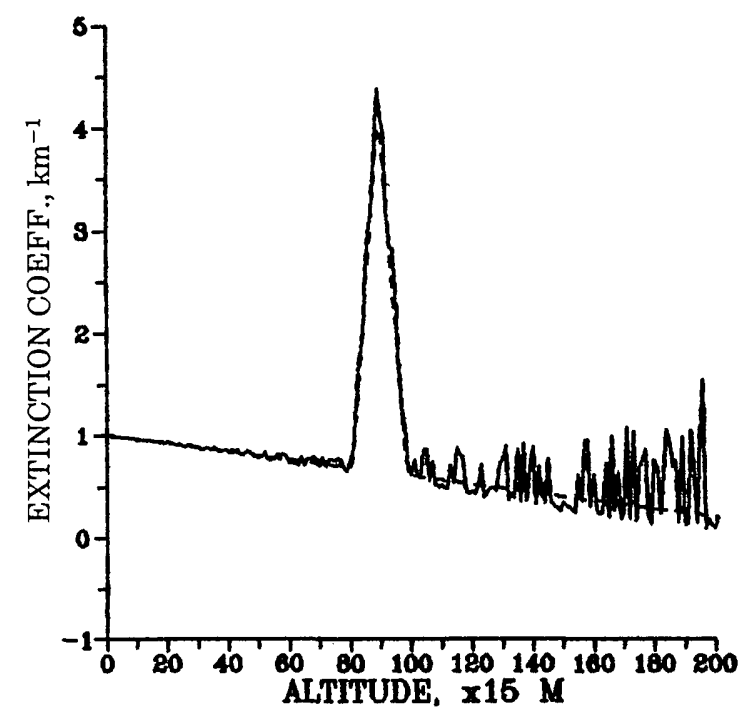

Fig. 2. Numerical experiment: the model extinction coefficient (dashed curve) and the derived Klett extinction coefficient (solid curve). 
nals, a simulated signal noise is added to the modeled $S$ functions, in accordance with the formula

$$
\begin{aligned}
& {\left[S_{N}(H)-S_{N}\left(H_{0}\right)\right]=S(H)-S\left(H_{0}\right)} \\
& \quad+\delta P_{\min }\left[1+\frac{1}{\left(H_{0} / H\right)\left\{\exp \left[S(H)-S\left(H_{0}\right)\right]\right\}}\right]
\end{aligned}
$$

where $\delta P_{\min }$ is the planned model error of the detected lidar signal in several initial space cells; $S_{N}(H)$ and $S_{N}\left(H_{0}\right)$, and $S(H)$ and $S\left(H_{0}\right)$, are either for $S_{\mathrm{RRS}}$ or for $S_{E}$. We choose $\delta P_{\min }=1 \%$ in the first 30 cells. In Eqs. (1)-(3) only the differences of the $S$ functions for the $H_{0}$ and the various $H$ are calculated, not the individual values. The model extinction is presented in Figs. 1 and 2 (by the dashed curve) together with

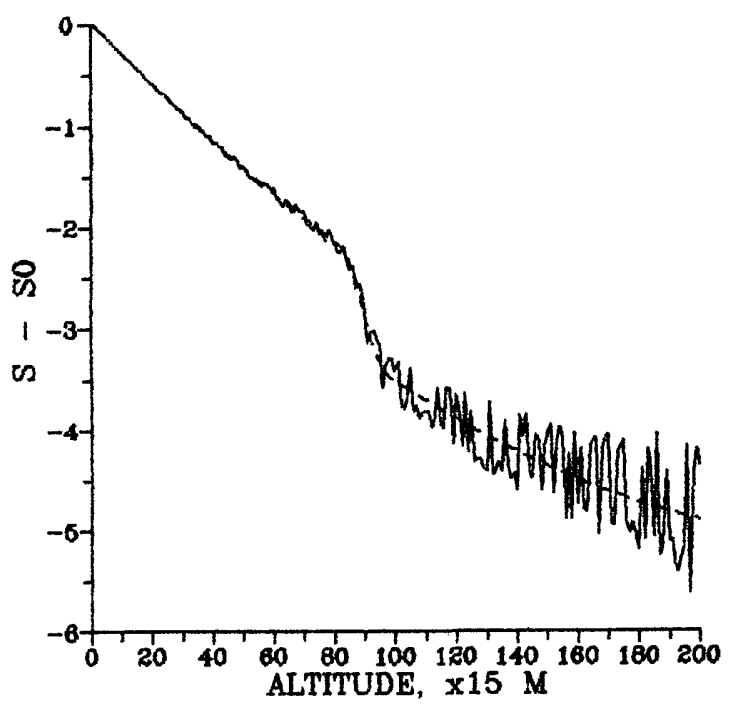

(a)

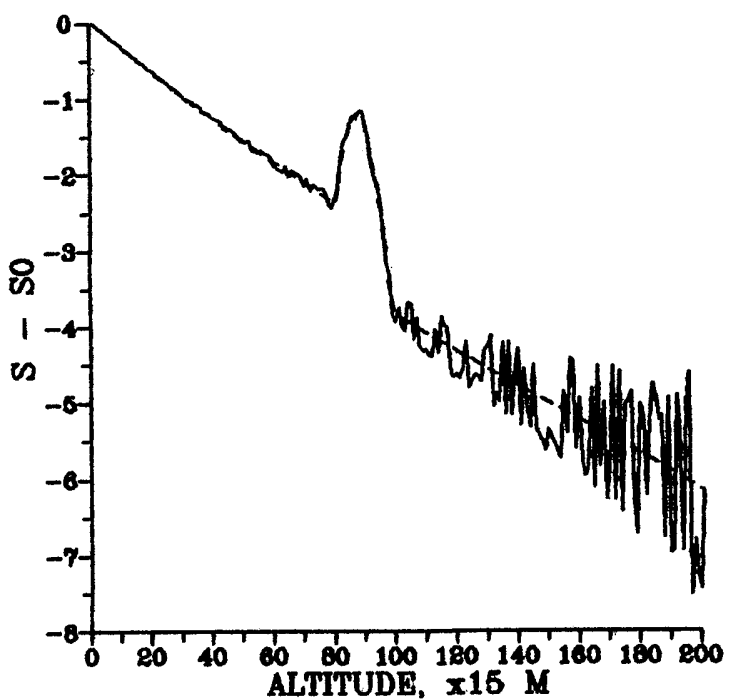

(b)

Fig. 3. (a) Numerical experiment: the rotational Raman backscatter normalized $S$ function $\left[S(H)-S\left(H_{0}\right)\right.$ ] without additive noise (dashed curve) and with additive noise (solid curve). (b) Numerical experiment: the elastic backscatter normalized $S$ function $\left[S(H)-S\left(H_{0}\right)\right]$ without additive noise (dashed curve) and with additive noise (solid curve). the derived log-derivative and Klett extinctions (solid curves). Figure 1(a) presents the log-derivative extinction with a twofold sliding average procedure for the extinction over five adjacent space cells. Figure 1(b) presents the log-derivative extinction with a tenfold sliding average. The Klett extinction coefficient profile in Fig. 2 is derived for $\alpha_{\text {ref }}=\alpha_{m}=0.2$ $\mathrm{km}^{-1}$, i.e., the boundary value of $\alpha_{\mathrm{ref}}$ is identical to the input model value $\alpha_{M}$. The normalized model $S$ functions without and with the additive noise are presented in Fig. 3(a) for the RRS lidar return and in Fig. 3(b) for the elastic lidar backscatter. The aerosol extinction coefficient is determined from the elastic and RRS return $S$ functions by using both the Klett and the RRS methods. As can be seen from Eq. (1) the log-derivative procedure is sensitive to small fluctuations of the RRS $S$ function. This takes place for the part of the profile beyond the model cloud layer and leads to a large deviation of the determined profile from the model profile for a low signal-to-noise ratio. Thus, in order to determine the extinction coefficient, we apply a moving average over five adjacent space samples: onefold for the RRS $S$ function and onefold or twofold for the determined log-derivative extinction coefficient profile. The determined log-derivative extinction profile is compared with the model one and with the Klett extinction profile. This comparison is based on regression analysis. The calculated statistical characteristics serve as criteria for establishing the certainty and the reliability of the employed mathematical procedures. Figure 4 presents the regression line of the log-derivative extinction coefficient $\alpha_{R}$ versus the model extinction coefficient $\alpha_{m}$. Figure 5 presents the regression line of $\alpha_{R}$ versus the Klett extinction $\alpha_{\mathrm{k}}$. The calculated values for $\alpha_{R}$, which are presented in Fig. 1(a) together with the model values $\alpha_{m}$, are employed in the regression analysis in Fig. 4.

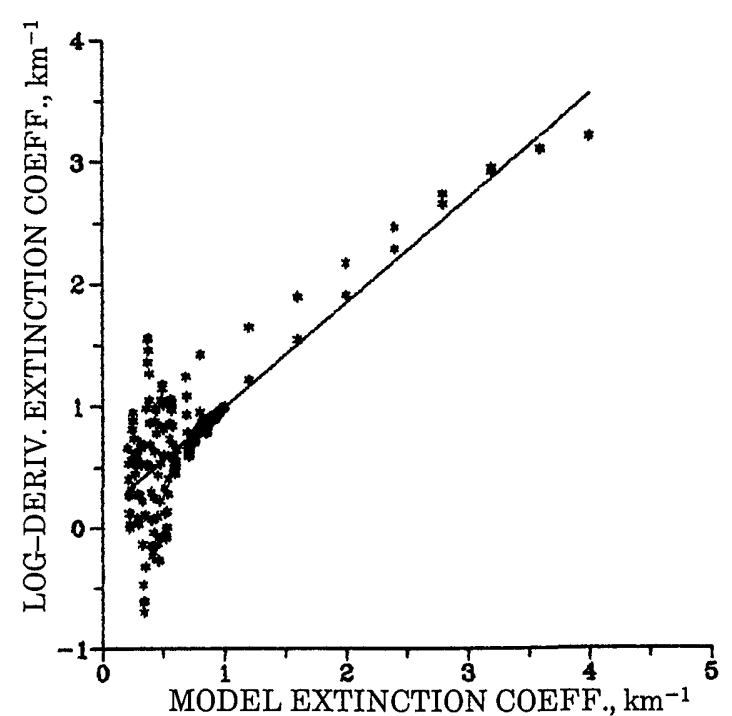

Fig. 4. Numerical experiment: log-derivative extinction coefficient and model extinction coefficient regression line. The data from Fig. 1(a) are used for the analysis. 


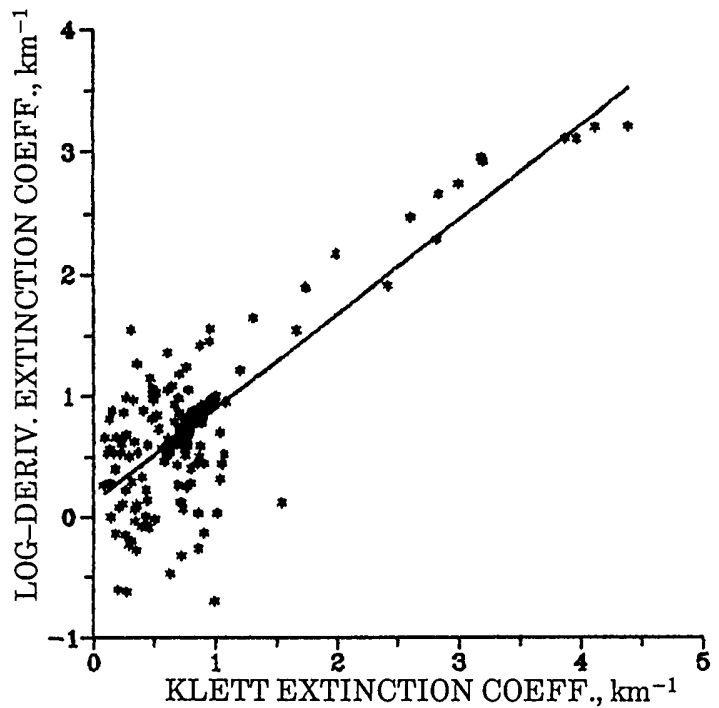

Fig. 5. Numerical experiment: log-derivative extinction coefficient and Klett extinction coefficient regression line. The values for the log-derivative extinction coefficient are those from Fig. 1(a) and those for the Klett extinction coefficient are from Fig. 2.

The same is true for $\alpha_{R}$ in Fig. 5, and the data for $\alpha_{\mathrm{k}}$ are those calculated values presented in Fig. 2.

The values of the statistical parameters of the regressions are given in Table 1 . The significance of the regression line coefficients, correlation coefficients $r$, and mean values $\bar{d}$ of the differences $\alpha_{\kappa}-\alpha_{R}$ or $\alpha_{m}-\alpha_{R}$, presented in Table 1, are determined by null-hypothesis verification procedures, ${ }^{6}$ which were carried out for each of these parameters. The parameters $F, t_{r}$, and $t_{d}$, which show the significance of the coefficients of regression, of the correlation coefficient, and of the mean value $\bar{d}$, respectively, are calculated (as in Ref. 6) for every particular regression. Their calculated values are compared with the tabulated values for $F$ (Fisher) and $t$ (Student) distribu- tions, $F_{\text {crit }}=254$ and $t_{\text {crit }}=1.96$, respectively, with an accepted probability of $95 \%$. The basic affirmation of the null hypothesis is that if $F<F_{\text {crit }}$ then the regression is insignificant and the slope of its line is near 0 . If $F>F_{\text {crit }}$ then the line of regression is significant with a probability of above $95 \%$. This is also analogous for $t_{r}$ and $t_{d}$.

The linear regression analysis of the model and the determined extinctions lead to the conclusion that the averaging that was used, onefold for the RRS $S$ function and twofold for the determined log-derivative extinction coefficient profile, is optimal. A confirmation of this is the increase in the significance of the regression lines and of the coefficients of correlation when multifold averaging is used. On the other hand, the additional, greater-than-twofold averaging of the log-derivative extinction profiles smooths the aerosol extinction layers, i.e., leads to loss of information for the aerosol distribution, and from that point of view is not optimal.

\section{Lidar Experimental Results}

The lidar setup is described in Ref. 4 . In the presented investigation the lidar was equipped with an additional detection channel to measure the aerosol backscattered signal profile. Below we give only the highlights of the system. The lidar has a coaxial optical structure. The telescope is a 14-in. Cassegrain type. The lidar makes use of a $\mathrm{CuBr}$-vapor laser with an average power of $2-3 \mathrm{~W}$ at the $510.6-\mathrm{nm}$ laser line and a beam divergency of 0.6-0.7 mrad. The laser pulse repetition rate is $16 \mathrm{kHz}$. A double polychromator is used to select two parts of the RRS of air for temperature profile measurements, ${ }^{6} \mathrm{RRS}$ extinction profile measurements obtained by using the log-derivative procedure ${ }^{4}$ and elastic backscattered radiation detection for subsequent implementation of the Klett inversion method. ${ }^{3}$ The detection of these three signals is carried out simultaneously by

Table 1. Characteristics of the Numerical Experiments and the Lidar Observation Limear Regression Lines ${ }^{a}$

\begin{tabular}{|c|c|c|c|c|c|}
\hline \multirow[b]{2}{*}{ Linear Regressions of } & \multicolumn{5}{|c|}{ Parameters of the Regressions } \\
\hline & $\begin{array}{l}\text { Parameter } F \\
\text { (Significance } \\
\text { of Regression) }\end{array}$ & $\begin{array}{c}\text { Correlation } \\
\text { Coefficient } \\
r\end{array}$ & $\begin{array}{l}\text { Parameter } t_{r} \\
\text { (Significance } \\
\text { of } r \text { ) }\end{array}$ & $\begin{array}{c}\text { Mean } \\
\text { Difference } \\
\overline{\bar{d}}\end{array}$ & $\begin{array}{l}\text { Parameter } t_{d} \\
\text { (Significance } \\
\text { of } \bar{d}\end{array}$ \\
\hline \multicolumn{6}{|l|}{ Numerical experiments } \\
\hline Twofold averaged $\alpha_{R}$ versus $\alpha_{m}, 200$ points & $\begin{array}{c}490 \\
\text { significant }\end{array}$ & $0.844 \pm 0.027$ & 22 & -0.023 & 0.97 \\
\hline Tenfold averaged $\alpha_{R}$ versus $\alpha_{m}, 200$ points & $\begin{array}{c}1056 \\
\text { significant }\end{array}$ & $0.918 \pm 0.027$ & 32 & -0.024 & 1.26 \\
\hline Twofold averaged $\alpha_{R}$ versus $\alpha_{K}, 200$ points & $\begin{array}{c}365 \\
\text { significant }\end{array}$ & $0.805 \pm 0.035$ & 19 & 0.061 & 2.16 \\
\hline Tenfold averaged $\alpha_{R}$ versus $\alpha_{K}, 200$ points & $\begin{array}{c}595 \\
\text { significant }\end{array}$ & $0.866 \pm 0.021$ & 24 & 0.064 & 2.61 \\
\hline \multicolumn{6}{|l|}{ Lidar measured $\alpha_{R}$ versus lidar measured $\alpha_{K}$} \\
\hline 20 October 1988,123 points, twofold averaged $\alpha_{R}$ & $\begin{array}{c}709 \\
\text { significant }\end{array}$ & $0.924 \pm 0.039$ & 27 & 0.022 & 0.37 \\
\hline 24 October 1988,200 points, twofold averaged $\alpha_{R}$ & $\begin{array}{c}50 \\
\text { insignificant }\end{array}$ & $0.451 \pm 0.042$ & 7 & 0.350 & 12 \\
\hline
\end{tabular}

${ }^{a} \alpha_{m}$ is the model, $\alpha_{R}$ is the log derivative, and $\alpha_{K}$ is the Klett extinction. 
three EMI 9789 QA photomultipliers in a photocounting mode. Data acquisition employs the buffer memory of an 8-bit personal computer that allows signal detection in 256 adjacent space samples with a $15-\mathrm{m}$ spatial resolution, and is started at the ground level by the laser pulse. Data storage proceeds independently for each of the three photomultipliers.

The lidar observations were carried out vertically during the night. The data-acquisition time for the presented lidar observations is $\sim 20 \mathrm{~min}$. For the log-derivative procedure the sum of the two RRS backscattered signals is used after corrections for the background noise and for the photomultipliers' afterpulses (signal-induced noise). The same corrections should be carried out on the elastic backscattered signal before applying the Klett inversion procedure. The reference extinction coefficient for this procedure is established by the least-squares linear approximation from the $S$ function value for the last five space cells. In the log-derivative procedure the altitude density distribution for standard atmosphere from Refs. 7 and 8 is used. There are systematic errors in the RRS extinction coefficient because of local temperature variations of the atmospheric density and, in a much lesser degree, because of the rotational Raman cross section. ${ }^{4}$ Because of these errors a simultaneous lidar measurement of the temperature profile is performed that makes use of the ratio of the two RRS backscattered signals. ${ }^{9}$

Examples of measurements of the atmospheric extinction coefficient profile are shown in Figs. 6 and 7. The linear regression of the RRS extinction coefficient versus the Klett extinction coefficient for these measurements is presented in Figs. 8 and 9. The results from the linear regression analysis for the lidar measurements are also presented in Table 1.

As is evident, the agreement between the log-

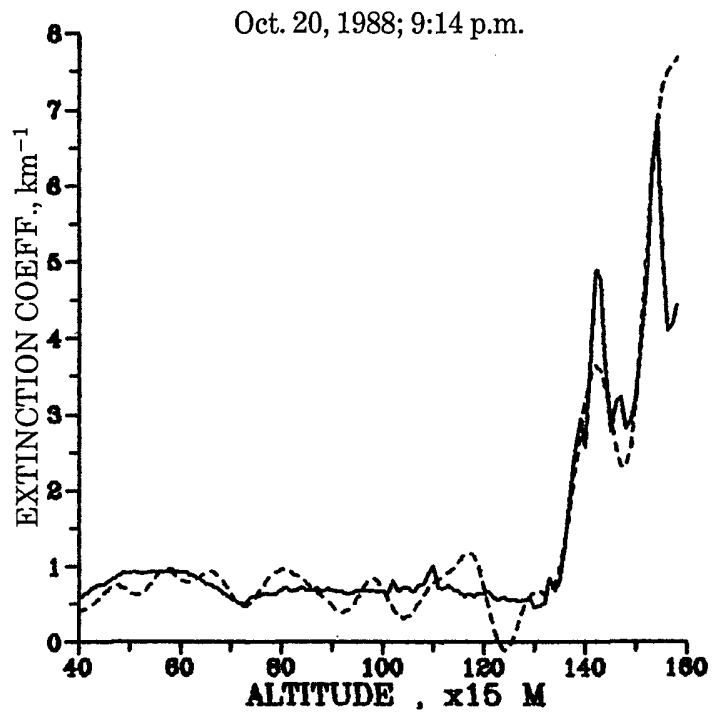

Fig. 6. Profiles made on 20 October 1988 for the Klett extinction coefficient (dashed curve) and for the log-derivative extinction coefficient (solid curve). The log-derivative extinction is twofold averaged.

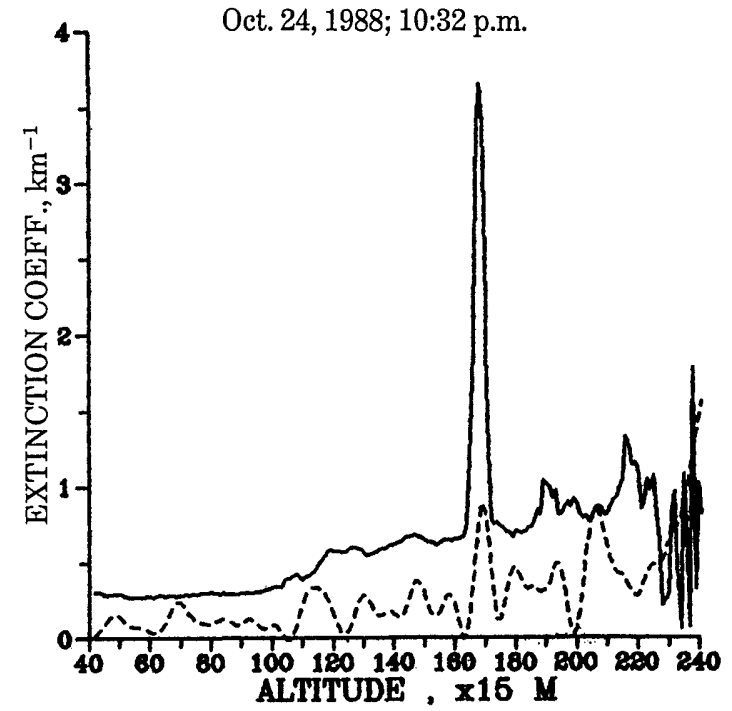

Fig. 7. Profiles made on 24 October 1988 for the Klett extinction coefficient (dashed curve) and for the log-derivative extinction coefficient (solid curve). The log-derivative extinction is twofold averaged.

derivative method and the Klett inversion method is much better for the measurement of 20 October 1988. In this case the optical thickness is larger and the Klett inversion works better. ${ }^{1,2}$ The agreement between the extinction profiles obtained by the two methods can be due to the specific meteorological conditions that permit the Klett inversion to give reasonable values to $\alpha_{\kappa}(H)$, although the applied constant lidar ratio is used. In addition, the calculated statistical parameters for this measurement, which are presented in Table 1, have values close to those of the modeled numerical experiments. Bearing this in mind, we consider that when the two methods are applied simultaneously, such an agree-

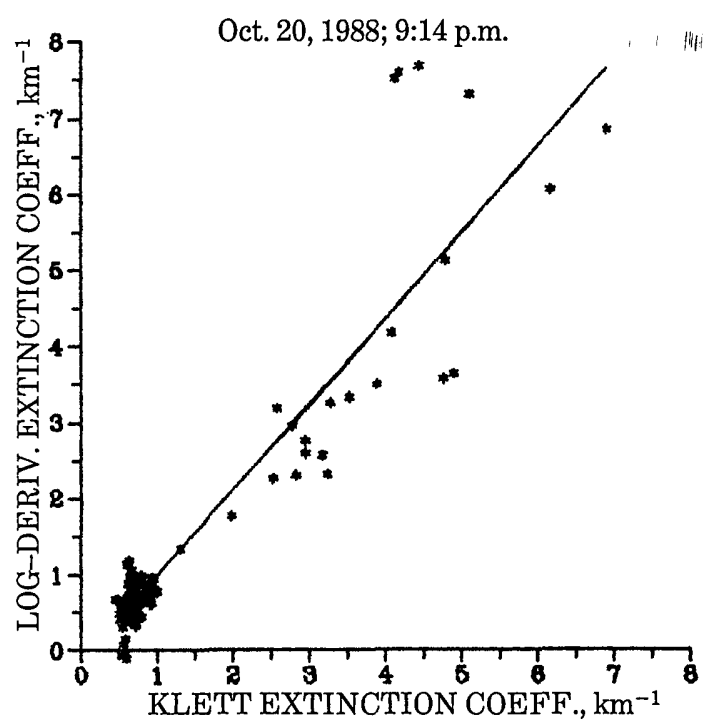

Fig. 8. Log-derivative extinction coefficient and Klett extinction coefficient regression line for the lidar measurement made on 20 October 1988. 


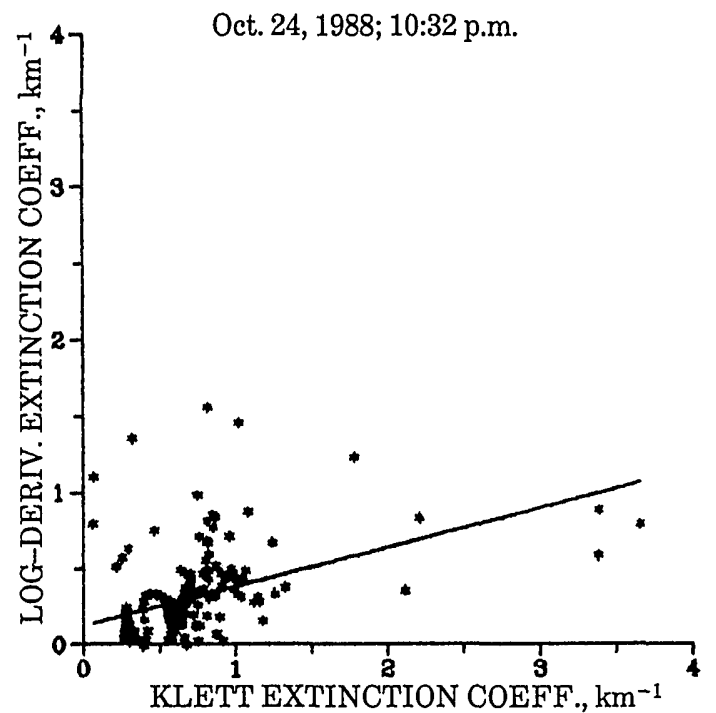

Fig. 9. Log-derivative extinction coefficient and Klett extinction coefficient regression line for the lidar measurement made on 24 October 1988.

ment can be used as verification of the reliability of the lidar measurements. In the absence of such an agreement, this reliability is under question and it is not clear which of the methods works better.

\section{Conclusion}

The presented results show that the advantages and setbacks of each method are complementary: a simplicity of the lidar and larger lidar signal, but a necessity for a priori information and assumptions for Klett method. On the other hand, there is no need for a priori information and assumptions for the log-derivative method but a much lower backscattered signal, with the technology presented above, restricts the lidar measurements to nighttime and requires a complicated lidar system.

From the above analysis of the numerical and lidar experiments, it follows that the comparison between the results of lidar extinction measurements on one hand and radiosonde or airborne visiometer measurements on the other, when used as criteria for the reliability of the lidar measurements, can be replaced with a comparison between the results of the two lidar methods. When a good agreement is found in such a comparison, we consider the values of the extinction profiles, measured with the lidar, to be close to the true ones.

We express our deep gratitude to our colleagues Y. F. Arshinov and S. M. Bobrovnikov of the Institute of Atmospheric Optics, Tomsk, Russia, for their suggestions and encouragement.

\section{References}

1. J. D. Klett, "Stable analytical inversion solution for processing lidar returns," Appl. Opt. 20, 211-220 (1981).

2. J. D. Klett, "Lidar inversion with variable backscatter/ extinction ratios," Appl. Opt. 24, 1638-1643 (1985).

3. I. Grigorov, V. Simeonov, P. Tomov, V. Mitev, Ya. Paneva, and P. Georgiev, "Experimental lidar determination of the extinction coefficient profile of visually clear atmosphere with low layer cloudiness," Bulg. J. Phys. 15, 173-181 (1988).

4. V. M. Mitev, I. V. Grigorov, V. B. Simeonov, Yu. F. Arshinov, and S. M. Bobrovnikov, "Raman lidar measurements of the atmospheric extinction coefficient profile," Bulg. J. Phys. 17, 67-74 (1990).

5. A. Ansmann, M. Riebesell, and C. Weitkamp, "Measurement of atmospheric aerosol extinction profiles with a Raman lidar," Opt. Lett. 15, 746-748 (1990).

6. J. H. Pollard, A Handbook of Numerical and Statistical Techniques (Cambridge U. Press, Cambridge, Mass., 1977), Chaps. 6-8.

7. I. V. Samokhvalov, ed., Spektroskopicheskiye Metodi Zondirovaniya Atmosfery (Spectroscopic Methods of Atmospheric Remote Sensing) (Nauka, Novosibirsk, 1985), pp. 30-39.

8. R. A. McClatchey, R. W. Fenn, and J. E. A. Selby, "Optical properties of the atmosphere," Rep. AFCRL-71-0279, Environmental Research Papers 354 (Air Force Cambridge Research Laboratories, Bedford, Mass., 1971), pp. 8-10.

9. Yu. F. Arshinov, S. M. Bobrovnikov, V. E. Zuev, and V. M. Mitev, "Atmospheric temperature measurements using a pure rotational Raman lidar," Appl. Opt. 22, 2984-2990 (1983). 